\title{
ON COUNTEREXAMPLES IN QUESTIONS OF UNIQUE DETERMINATION OF CONVEX BODIES
}

\author{
DMITRY RYABOGIN AND VLAD YASKIN
}

(Communicated by Thomas Schlumprecht)

\begin{abstract}
We discuss a construction that gives counterexamples to various questions of unique determination of convex bodies.
\end{abstract}

\section{INTRODUCTION}

This note is motivated by the following question from the book Geometric Tomography by R. J. Gardner [G, Prob. 7.6, p. 289].

Question 1.1. Let $K$ and $L$ be origin-symmetric convex bodies in $\mathbb{R}^{3}$ such that the sections $K \cap H$ and $L \cap H$ have equal perimeters for every 2-dimensional subspace $H$ of $\mathbb{R}^{3}$. Is it true that $K=L$ ?

The problem has attracted a lot of attention, but at the moment it is still open; see [HNRZ, $[\mathrm{R},[\mathrm{Y}]$ for some partial results. The question can also be generalized to higher dimensions and all intermediate intrinsic volumes $V_{i}$, as well as sections of other dimensions.

In this note we show that the question has a negative answer in the class of general (not necessarily symmetric) convex bodies.

One of the natural steps in generalizing questions involving sections is to replace sections by slabs (or "thick sections"). We discuss such problems at the end of the paper.

Throughout the paper we use the following notation. We write $S^{n-1}$ and $B_{2}^{n}$ for the unit sphere and closed unit ball in Euclidean space $\mathbb{R}^{n}$, and $G(n, k)$ for the Grassmannian of all $k$-dimensional subspaces of $\mathbb{R}^{n}$. The hyperplane through the origin with normal vector $\xi \in S^{n-1}$ is denoted by $\xi^{\perp}$. A convex body in $\mathbb{R}^{n}$ is a compact convex set with nonempty interior. In this paper we always assume that convex bodies contain the origin in their interiors. The orthogonal projection of a convex body $K$ onto a subspace $V$ is denoted by $K \mid V$. If $Q$ is a convex body in an $i$-dimensional subspace $V \subseteq \mathbb{R}^{n}, 1 \leq i \leq n$, then we write $\operatorname{vol}_{i}(Q)$ for its volume. For a set $C$ in $\mathbb{R}^{n}$, its reflection in the origin is denoted by $-C$. We say that a set $C$ is origin symmetric if $C=-C$. Two sets $C$ and $D$ in $\mathbb{R}^{n}$ are said to be congruent if there is an isometry $T$ of $\mathbb{R}^{n}$ such that $T(C)=D$.

Received by the editors September 13, 2011 and, in revised form, October 10, 2011; October 21, 2011; and November 8, 2011.

2010 Mathematics Subject Classification. Primary 52A20.

Key words and phrases. Convex bodies, sections, projections, slabs, intrinsic volumes.

The first author was supported in part by U.S. National Science Foundation Grants DMS0652684 and DMS-1101636.

The second author was supported in part by NSERC. 
For a convex body $K$ in $\mathbb{R}^{n}$ its support function $h_{K}$ is defined by

$$
h_{K}(x)=\max \{\langle x, y\rangle: y \in K\}, \quad x \in \mathbb{R}^{n} .
$$

Clearly, $h_{K}$ is positively homogeneous of degree 1 and therefore is determined by its values on the unit sphere. It is also easy to see that for any subspace $V$ of $\mathbb{R}^{n}$ the support function of $K \mid V$, as a convex body in $V$, is just the restriction of $h_{K}$ to $V$.

If $K$ is a compact set containing the origin in its interior and star-shaped with respect to the origin, then its radial function $\rho_{K}$ is defined by

$$
\rho_{K}(x)=\max \{\lambda>0: \lambda x \in K\}, \quad x \in S^{n-1} .
$$

We say that a convex body $K$ is of class $C_{+}^{\infty}$ if its boundary is a $C^{\infty}$ manifold in $\mathbb{R}^{n}$ with positive Gaussian curvature at each point.

Let $K$ be a compact convex set in $\mathbb{R}^{n}$. Its intrinsic volumes $V_{i}(K), 1 \leq i \leq n$, can be defined via Steiner's formula

$$
\operatorname{vol}_{n}\left(K+\epsilon B_{2}^{n}\right)=\sum_{i=0}^{n} \kappa_{n-i} V_{i}(K) \epsilon^{n-i},
$$

where the addition is the Minkowski addition, $\kappa_{n-i}$ is the volume of the $(n-i)$ dimensional Euclidean ball, and $\epsilon \geq 0$.

In particular, if $K$ is a convex body in $\mathbb{R}^{n}$, then $V_{n}(K)$ is its volume and $V_{n-1}(K)$ is half the surface area. For these and other facts about intrinsic volumes we refer the reader to the book $[\underline{\mathrm{S}}$.

\section{Results}

The idea of the construction that we use in this note goes back to Gardner and Volčič GV] and to Goodey, Schneider and Weil GSW].

First we prove an auxiliary lemma.

Lemma 2.1. Let $K$ and $L$ be convex bodies in $\mathbb{R}^{n}$ containing the origin in their interiors. The following properties are equivalent:

i) $K \backslash L=-(L \backslash K)$,

ii) $\left\{h_{K}(\xi), h_{K}(-\xi)\right\}=\left\{h_{L}(\xi), h_{L}(-\xi)\right\}$, for all $\xi \in S^{n-1}$,

iii) $\left\{\rho_{K}(\xi), \rho_{K}(-\xi)\right\}=\left\{\rho_{L}(\xi), \rho_{L}(-\xi)\right\}$, for all $\xi \in S^{n-1}$.

Proof. The equivalence of (i) and (ii) is shown in GSW.

Let us show that (iii) implies (i). Let $x \in K \backslash L$. Since $K$ and $L$ are star-shaped with respect to the origin, $\rho_{K}(x /|x|)>\rho_{L}(x /|x|)$. Now (iii) yields $\rho_{K}(x /|x|)=$ $\rho_{L}(-x /|x|)$ and $\rho_{K}(-x /|x|)=\rho_{L}(x /|x|)$. Therefore, $\rho_{K}(-x /|x|)<\rho_{L}(-x /|x|)$, i.e. $x \in-(L \backslash K)$. We have shown that $K \backslash L \subset-(L \backslash K)$. The other inclusion is obtained in a similar fashion. Thus, $K \backslash L=-(L \backslash K)$.

It remains to show that (i) implies (iii). Let $\xi \in S^{n-1}$. Consider two cases: $\rho_{K}(\xi)=\rho_{L}(\xi)$ and $\rho_{K}(\xi) \neq \rho_{L}(\xi)$. In the first case we claim that $\rho_{K}(-\xi)=$ $\rho_{L}(-\xi)$. Indeed, if the latter is not true, then there exists $x$ on the boundary of $K$ (say) such that $x /|x|=-\xi$ and $\rho_{K}(-\xi)>\rho_{L}(-\xi)$. But then $-x \in L \backslash K$, meaning that $\rho_{K}(\xi)<\rho_{L}(\xi)$. We reach a contradiction.

In the second case, let $\rho_{K}(\xi)>\rho_{L}(\xi)$, say. Consider the ray $\left\{x \in \mathbb{R}^{n}: x=t \xi, t \geq\right.$ $0\}$. It intersects the boundaries of $L$ and $K$ at some points, which we will denote by $p$ and $q$ correspondingly. Since $K \backslash L=-(L \backslash K)$, the ray $\left\{x \in \mathbb{R}^{n}: x=-t \xi, t \geq 0\right\}$ intersects $L \backslash K$ in the interval $(-p,-q]$. Hence, the points $-p$ and $-q$ are on the 
boundaries of $L$ and $K$ correspondingly. This implies that $\rho_{K}(\xi)=\rho_{L}(-\xi)$ and $\rho_{K}(-\xi)=\rho_{L}(\xi)$.

For the reader's convenience, we provide a short proof of the following proposition. Examples of this type appeared in G, Thm. 3.3.17, Thm. 3.3.18] (see also GV]).

Proposition 2.2. There exist noncongruent convex bodies $K$ and $L$ satisfying the properties of Lemma 2.1. Moreover, the bodies can be chosen to be $C_{+}^{\infty}$ bodies of revolution or polytopes.

Proof. 1) $K$ and $L$ are $C_{+}^{\infty}$ bodies of revolution.

We start with two nonzero infinitely smooth functions $\phi$ and $\psi$, supported in the intervals $(1 / 3-\delta, 1 / 3+\delta)$ and $(2 / 3-\delta, 2 / 3+\delta)$ correspondingly, where $0<\delta<1 / 6$. Next, for $t \in[-1,1]$ and $\epsilon>0$, we define

$$
\begin{gathered}
f(t)=\sqrt{1-t^{2}}+\epsilon \phi(t)+\epsilon \psi(t), \\
g(t)=\sqrt{1-t^{2}}+\epsilon \phi(t)+\epsilon \psi(-t) .
\end{gathered}
$$

Finally, we choose $\epsilon$ sufficiently small to guarantee that $f$ and $g$ are concave on $[-1,1]$.

Now we consider two convex bodies in $\mathbb{R}^{n}$,

$K=\left\{x \in \mathbb{R}^{n}: x_{1}^{2}+\cdots+x_{n-1}^{2} \leq f^{2}\left(x_{n}\right)\right\}$ and $L=\left\{x \in \mathbb{R}^{n}: x_{1}^{2}+\cdots+x_{n-1}^{2} \leq g^{2}\left(x_{n}\right)\right\}$.

The boundaries of $K$ and $L$ are generated by rotating the graphs of the functions $f$ and $g$ defined above. Since these generating curves are not congruent, it follows that $K$ and $L$ are not congruent.

By construction, $K$ and $L$ are of class $C^{\infty}$, and also $K \backslash L=-(L \backslash K)$. Finally, choosing $\epsilon>0$ sufficiently small, $\left\|\rho_{K}-\rho_{B_{2}^{n}}\right\|_{C^{2}\left(S^{n-1}\right)}$ and $\left\|\rho_{L}-\rho_{B_{2}^{n}}\right\|_{C^{2}\left(S^{n-1}\right)}$ can be made as small as we wish. By formula (0.41) from $G$ ] this is enough to ensure that $K$ and $L$ have strictly positive curvature.

2) $K$ and $L$ are polytopes.

Let $a_{1}, \ldots, a_{n}$ be distinct positive numbers and consider the rectangular box $R=$ $\left[-a_{1}, a_{1}\right] \times \cdots \times\left[-a_{n}, a_{n}\right]$. Take any two vertices $u$ and $v$ of $R$ that are connected by an edge. The idea is that $K$ will be constructed by cutting off vertices $u$ and $v$ from $R$, and $L$ by cutting off vertices $u$ and $-v$.

More precisely, let $F$ and $H$ be supporting hyperplanes to $R$ at the vertices $u$ and $v$ correspondingly, such that $R \cap F=\{u\}$ and $R \cap H=\{v\}$. Suppose that $F$ is given by $\langle x, \xi\rangle=t$ for some $\xi \in S^{n-1}$ and $t>0$ and that $H$ is given by $\langle x, \eta\rangle=s$ for some $\eta \in S^{n-1}$ and $s>0$. If $\lambda>0$ is sufficiently small, then the sets $R \cap\{x:\langle x, \xi\rangle \geq t-\lambda\}$ and $R \cap\{x:\langle x, \eta\rangle \geq s-\lambda\}$ do not overlap and contain no vertices of $R$ other than $u$ and $v$ correspondingly. Define

$$
\begin{gathered}
K=R \cap\{x:\langle x, \xi\rangle \leq t-\lambda\} \cap\{x:\langle x, \eta\rangle \leq s-\lambda\}, \\
L=R \cap\{x:\langle x, \xi\rangle \leq t-\lambda\} \cap\{x:\langle x, \eta\rangle \geq-s+\lambda\} .
\end{gathered}
$$

The bodies $K$ and $L$ are not congruent, since the vertices $u$ and $v$ that we cut off from $R$ belong to the same edge, while this is not true for $u$ and $-v$.

Finally, observe that $K$ and $L$ satisfy the property (i) of Lemma 2.1 .

Lemma 2.1 and Proposition 2.2 lead to the following result; see Theorems 3.3.17, $3.3 .18,6.2 .18$, and 6.2 .19 in $\mathrm{G}$. 
Corollary 2.3. There are noncongruent convex bodies $K$ and $L$ in $\mathbb{R}^{n}$ such that $\forall \xi \in S^{n-1}$,

$$
\operatorname{vol}_{n-1}\left(K \mid \xi^{\perp}\right)=\operatorname{vol}_{n-1}\left(L \mid \xi^{\perp}\right)
$$

and

$$
\operatorname{vol}_{n-1}\left(K \cap \xi^{\perp}\right)=\operatorname{vol}_{n-1}\left(L \cap \xi^{\perp}\right) .
$$

This result remains true if the $(n-1)$-dimensional volume of the projections is replaced by their $i$-th intrinsic volumes. See Gardner [G, Thm. 3.3.17, Thm. 3.3.18] for $i=1, n-1$, and Goodey, Schneider and Weil [GSW] for all other cases.

Now we return to Question 1.1 stated in the introduction. The next theorem shows that without the assumption of origin-symmetry, this question would have a negative answer.

Theorem 2.4. There are noncongruent convex bodies $K$ and $L$ in $\mathbb{R}^{n}$ such that for all $i$ and $k$ with $1 \leq i \leq k \leq n-1$ we have

$$
V_{i}(K \cap H)=V_{i}(L \cap H)
$$

for all $H \in G(n, k)$.

Moreover, $K$ and $L$ can be constructed in such a way that both of them are $C_{+}^{\infty}$ bodies of revolution or both $K$ and $L$ are polytopes.

Proof. Let $K$ and $L$ be noncongruent convex bodies in $\mathbb{R}^{n}$ such that

$$
\left\{\rho_{K}(\xi), \rho_{K}(-\xi)\right\}=\left\{\rho_{L}(\xi), \rho_{L}(-\xi)\right\},
$$

for all $\xi \in S^{n-1}$. Recall that $C_{+}^{\infty}$ and polytopal examples of such bodies were given in Proposition 2.2 .

Then, for every $H \in G(n, k)$, the convex bodies $K \cap H$ and $L \cap H$ in the subspace $H$ satisfy the relation

$$
\left\{\rho_{K \cap H}(\xi), \rho_{K \cap H}(-\xi)\right\}=\left\{\rho_{L \cap H}(\xi), \rho_{L \cap H}(-\xi)\right\}, \quad \forall \xi \in S^{n-1} \cap H .
$$

By Lemma 2.1, for these $k$-dimensional bodies we also have

$$
\left\{h_{K \cap H}(\xi), h_{K \cap H}(-\xi)\right\}=\left\{h_{L \cap H}(\xi), h_{L \cap H}(-\xi)\right\}, \quad \forall \xi \in S^{n-1} \cap H,
$$

and, in particular, the last relation is true for the projections of $K \cap H$ and $L \cap$ $H$ onto $i$-dimensional subspaces of $H, 1 \leq i \leq k$. We apply Lemma 2.1 to get $\operatorname{vol}_{i}((K \cap H) \mid V)=\operatorname{vol}_{i}((L \cap H) \mid V)$ for every $i$-dimensional subspace $V$ of $H$.

By Kubota's integral recursion (see [S, p. 295]), we have

$$
V_{i}(K \cap H)=c_{i, k} \int_{G(H, i)} \operatorname{vol}_{i}((K \cap H) \mid V) d V,
$$

for some constant $c_{i, k}$. Here, by $G(H, i)$ we mean the Grassmannian of all $i$-dimensional subspaces of $H$. Thus, $V_{i}(K \cap H)=V_{i}(K \cap H)$.

The following result is proved in $[\mathrm{Y}]$. Let $k$ be an integer with $2 \leq k \leq n-1$. If $K$ and $L$ are origin-symmetric convex polytopes in $\mathbb{R}^{n}$ such that $V_{k-1}(K \cap H)=$ $V_{k-1}(K \cap H)$ for all $H \in G(n, k)$, then $K=L$. Theorem 2.4 shows that this result is false without the symmetry assumption.

It is interesting to note that the following result is true. 
Proposition 2.5. Let $i \neq j$ be integers, $1 \leq i, j \leq n-1$, and let $K$ and $L$ be convex bodies (containing the origin in their interiors) such that

$$
\operatorname{vol}_{i}(K \cap V)=\operatorname{vol}_{i}(L \cap V), \quad \forall V \in G(n, i),
$$

and

$$
\operatorname{vol}_{j}(K \cap W)=\operatorname{vol}_{j}(L \cap W), \quad \forall W \in G(n, j) .
$$

Then for all $k, 1 \leq k \leq n-1$, we have

$$
\operatorname{vol}_{k}(K \mid H)=\operatorname{vol}_{k}(L \mid H), \quad \forall H \in G(n, k) .
$$

Proof. Conditions (11) and (2) yield that $\left\{\rho_{K}(\xi), \rho_{K}(-\xi)\right\}=\left\{\rho_{L}(\xi), \rho_{L}(-\xi)\right\}$, for all $\xi \in S^{n-1}$; see G, Thm. 7.2.3, Thm. 6.2.16]. By Lemma 2.1, this is equivalent to the condition $\left\{h_{K}(\xi), h_{K}(-\xi)\right\}=\left\{h_{L}(\xi), h_{L}(-\xi)\right\}$, for all $\xi \in S^{n-1}$. As shown in [GSW], this implies (3).

Clearly, if $K$ and $L$ are two convex bodies such that all their corresponding $i$-dimensional projections have the same $i$-volume and all corresponding $j$ dimensional projections have the same $j$-volume, then one cannot expect that the sections of $K$ and $L$ will have equal volumes (simply because sections are not invariant under translations). Under the same conditions, is it possible to translate $K$ and $L$ so that all their sections would have the same volumes? The answer is again negative. Goodey, Schneider and Weil [GSW, p. 86] remark that there are convex polytopes $K$ and $L$ such that (3) holds for all $k$, but none of their translates satisfy $K \backslash L=-(L \backslash K)$. Thus, as the proof of Proposition 2.5] shows, no translates of $K$ and $L$ can satisfy (11) and (2) for some $i \neq j$.

Our next question concerns slabs in convex bodies. For $t>0$ and $\xi \in S^{n-1}$ consider the slab of width $2 t$ perpendicular to $\xi$ defined by

$$
S_{t}(\xi)=\left\{x \in \mathbb{R}^{n}:|\langle x, \xi\rangle| \leq t\right\} .
$$

There are various problems related to volumes of slabs in convex bodies; see e.g. $[\mathrm{BK}], \mathrm{KK}]$. Here we suggest the following.

Question 2.6. Let $K$ and $L$ be origin-symmetric convex bodies in $\mathbb{R}^{n}$ that contain the Euclidean ball of radius $t$ in their interiors. Suppose that for some $i(1 \leq i \leq n)$

$$
V_{i}\left(K \cap S_{t}(\xi)\right)=V_{i}\left(L \cap S_{t}(\xi)\right), \quad \forall \xi \in S^{n-1} .
$$

Is it true that $K=L$ ?

Our next result shows that the previous question has a negative answer if we drop the assumption of origin-symmetry.

Theorem 2.7. There are noncongruent convex bodies $K$ and $L$ in $\mathbb{R}^{n}$ that contain the Euclidean ball of radius $t>0$ in their interiors and such that

$$
V_{i}\left(K \cap S_{t}(\xi)\right)=V_{i}\left(L \cap S_{t}(\xi)\right), \quad \forall \xi \in S^{n-1},
$$

for all $1 \leq i \leq n$.

Moreover, both $K$ and $L$ can be chosen to be $C_{+}^{\infty}$ bodies of revolution or polytopes, as in Theorem 2.4.

Proof. Consider the same pair of bodies $K$ and $L$ as used in Theorem 2.4. Without loss of generality, we may assume that $t>0$ is small enough so that $t B_{2}^{n}$ is contained in the interior of both $K$ and $L$. Since $S_{t}(\xi)=-S_{t}(\xi)$, it follows that

$$
\left\{\rho_{K \cap S}(\theta), \rho_{K \cap S}(-\theta)\right\}=\left\{\rho_{L \cap S}(\theta), \rho_{L \cap S}(-\theta)\right\}, \quad \forall \theta \in S^{n-1},
$$


where for brevity we write $S=S_{t}(\xi)$. Now, as in Theorem 2.4, we see that all $i$-dimensional projections of $K \cap S$ and $L \cap S$ have the same $i$-volumes, and Kubota's integral recursion finishes the proof.

Since intrinsic volumes are continuous (in the Hausdorff metric) valuations on convex sets, it follows that Theorem 2.4 can be derived from Theorem 2.7. It is enough to notice that $K \cap S_{t}(\xi)$ approaches $K \cap \xi^{\perp}$ in the Hausdorff metric.

\section{REFERENCES}

[BK] F. Barthe, A. Koldobsky, Extremal slabs in the cube and the Laplace transform, Adv. Math. 174 (2003), 89-114. MR1959893 (2004f:52004)

[G] R.J. Gardner, Geometric tomography, Second edition. Encyclopedia of Mathematics and its Applications, 58. Cambridge University Press, New York, 2006. MR 2251886 (2007i:52010)

[GV] R.J. GaRdner, A. VolČIČ, Tomography of convex and star bodies, Adv. Math. 108 (1994), 367-399. MR1296519(95j:52013)

[GSW] P. GoOdey, R. SchNEIDER AND W. WeIL, On the determination of convex bodies by projection functions, Bull. London Math. Soc. 29 (1997), 82-88. MR.1416411 (97g:52017)

[HNRZ] R. Howard, F. Nazarov, D. Ryabogin and A. Zvavitch, Determining starlike bodies by the perimeters of their central sections, preprint.

[KK] A. Koldobsky, H. KÖNIG, Volumes of lower-dimensional slabs and sections of the cube, Adv. Appl. Math. 47 (2011), 894-907. MR2832384

[R] A. Rusu, Determining starlike bodies by their curvature integrals, Ph.D. Thesis, University of South Carolina, 2008. MR2711955

[S] R. Schneider, Convex Bodies: The Brunn-Minkowski Theory, Cambridge University Press, Cambridge, 1993. MR1216521 (94d:52007)

[Y] V. YAskin, On perimeters of sections of convex polytopes, J. Math. Anal. Appl. 371 (2010), 447-453. MR2670125(2011h:52017)

Department of Mathematics, Kent State University, Kent, Ohio 44242

E-mail address: ryabogin@math.kent.edu

Department of Mathematical and Statistical Sciences, University of Alberta, Edmonton, Alberta T6G 2G1, Canada

E-mail address: vladyaskin@math.ualberta.ca 